Kragujevac Journal of Mathematics

Volume 45(3) (2021), Pages 449-463.

\title{
OPTIMIZATIONS ON STATISTICAL HYPERSURFACES WITH CASORATI CURVATURES
}

\author{
ALIYA NAAZ SIDDIQUI ${ }^{1}$ AND MOHAMMAD HASAN SHAHID ${ }^{1}$
}

\begin{abstract}
In the present paper, we study Casorati curvatures for statistical hypersurfaces. We show that the normalized scalar curvature for any real hypersurface (i.e., statistical hypersurface) of a holomorphic statistical manifold of constant holomorphic sectional curvature $k$ is bounded above by the generalized normalized $\delta$-Casorati curvatures and also consider the equality case of the inequality. Some immediate applications are discussed.
\end{abstract}

\section{INTRODUCTION}

In 1985, a notion of statistical manifold has been studied by Amari [1]. The abstract generalizations of statistical models are considered as the statistical manifolds. The geometry of statistical manifolds lies at a junction of several branches of geometry (information geometry, affine differential geometry and Hessian geometry). A statistical structure can be considered as a generalization of a Riemannian structure (a pair of a Riemannian metric and its Levi-Civita connection). It includes the notion of dual connection, also called conjugate connection. The theory of statistical manifold and its statistical submanifold plays a role of central importance in many research fields of differential geometry.

Recently, H. Furuhata investigated the existence of complex structures on statistical manifolds and introduced the concept of holomorphic statistical manifold, as the statistical counterpart of the notion of complex manifold (see [11,12]). Similarly, by putting a natural affine connection to a Sasakian manifold and a Kenmotsu manifold, Furuhata defined a Sasakian statistical manifold [13] and a Kenmotsu statistical

Key words and phrases. $\delta$-Casorati curvatures, holomorphic statistical manifold, statistical hypersurfaces, normalized scalar curvature, dual connections.

2010 Mathematics Subject Classification. Primary: 53C05. Secondary: 49K35, 62B10.

DOI 10.46793/KgJMat2103.449S

Received: June 23, 2018.

Accepted: February 18, 2019. 
manifold [14]. The theory of statistical manifolds and their statistical submanifolds is a very recent geometry. Therefore, it attracts the geometers and several interesting results have been obtained by many of them (for example [3-5, 21, 22, 26, 28]).

The Casorati curvature has been defined by F. Casorati [6] as the normalized square of the length of the second fundamental form of a submanifold of a Riemannian manifold. This notion extends the concept of the principal direction of a hypersurface of a Riemannian manifold. This curvature, which is of interest in computer vision, was preferred by Casorati over the traditional curvatures because it seems to correspond better with the common intuition of curvature. Several geometers have found geometrical interpretation and significance of the (extrinsic) Casorati curvatures. Therefore, it follows that it is of great interest to establish a family of optimal Casorati inequalities for different submanifolds with any codimension of different ambient space forms (for example $[9,10,15,16,18,19,24,25,27]$ )

In this paper, we obtain a family of optimal inequalities which relate the normalized scalar curvature with the Casorati curvature for statistical hypersurfaces of holomorphic statistical manifolds of constant holomorphic sectional curvature. Equality cases are also verified. Such inequalities were recently obtained for a statistical submanifold, which is obviously a particular class of statistical hypersurfaces. See, for instance $[2,8,17,20]$. We mention that the ambient spaces in the above mentioned articles are different as compared to the ambient space (that is, a holomorphic statistical manifold of constant holomorphic sectional curvature) in our work, namely a quaternion Kahler-like statistical space form, a Kenmotsu statistical manifold, a statistical manifold, and a Sasakian statistical manifold, respectively.

\section{Statistical Manifold and its Submanifolds}

This section is fully devoted to a brief review of several fundamental formulae and some definitions which are required later.

Definition 2.1 ([12]). Let $\bar{\nabla}$ be an affine connection of Riemannian manifold $(\overline{\mathcal{M}}, \bar{g})$ with Riemannian metric $\bar{g}$ on $\overline{\mathcal{M}}$.

(a) The affine connection $\bar{\nabla}^{*}$ of $\overline{\mathcal{M}}$ defined by

$$
z \bar{g}(x, y)=\bar{g}\left(\bar{\nabla}_{z} x, y\right)+\bar{g}\left(x, \bar{\nabla}_{z}^{*} y\right)
$$

for any $\mathcal{X}, y, z \in \Gamma(T \overline{\mathcal{M}})$ is known as the dual connection of $\bar{\nabla}$ with respect to $\bar{g}$.

(b) The triplet $(\overline{\mathcal{M}}, \bar{\nabla}, \bar{g})$ is known as a statistical manifold if the torsion tensor field of $\bar{\nabla}$ vanishes and $\bar{\nabla} \bar{g} \in \Gamma\left(T \overline{\mathcal{M}}^{(0,3)}\right)$ is symmetric.

Remark 2.1. If $(\overline{\mathcal{M}}, \bar{\nabla}, \bar{g})$ is a statistical manifold, so is $\left(\overline{\mathcal{M}}, \bar{\nabla}^{*}, \bar{g}\right)$. The dual connections $\bar{\nabla}$ and $\bar{\nabla}^{*}$ of $\overline{\mathcal{M}}$ satisfy (see [12]) $\left(\bar{\nabla}^{*}\right)^{*}=\bar{\nabla}$ and $2 \bar{\nabla}^{0}=\bar{\nabla}+\bar{\nabla}^{*}$, where $\bar{\nabla}^{0}$ is Levi-Civita connection for $\overline{\mathcal{M}}$ of $\bar{g}$. 
Example 2.1. Let $(\overline{\mathcal{M}}, \bar{g})$ be a family of exponential distributions of mean 0

$$
\overline{\mathcal{M}}:=\left\{p(u, \Phi) \mid p(u, \Phi)=\Phi e^{-\Phi u}, u \in[0, \infty), \Phi \in(0, \infty)\right\},
$$

a Riemannian metric is given by $\bar{g}:=\Phi^{-2}(d \Phi)^{2}$, and an $\alpha$-connection $(\alpha \in \mathbb{R})$ on $\overline{\mathcal{M}}$ is defined by

$$
\bar{\nabla}_{\frac{\partial}{\partial \Phi}}^{\alpha} \frac{\partial}{\partial \Phi}=(\alpha-1) \Phi^{-1} \frac{\partial}{\partial \Phi}
$$

Then, $\left(\overline{\mathcal{M}}, \bar{\nabla}^{\alpha}, \bar{g}\right)$ is a 1-dimensional statistical manifold.

We remark that one can also construct examples for higher dimension by defining Fisher information metric and $\alpha$-connection on a family of statistical distribution (for example [12]).

Definition $2.2([12])$. Let $(\overline{\mathcal{N}}, \bar{\nabla}, \bar{g})$ be a statistical manifold and $\mathcal{M}$ be a submanifold of $\overline{\mathcal{M}}$. Then $(\mathcal{M}, \nabla, g)$ is also a statistical manifold with the induced statistical structure $(\nabla, g)$ on $\mathcal{M}$ from $(\bar{\nabla}, \bar{g})$ and we call $(\mathcal{M}, \nabla, g)$ as a statistical submanifold in $(\overline{\mathcal{M}}, \bar{\nabla}, \bar{g})$.

Definition $2.3([12])$. Let $(\overline{\mathcal{M}}, \bar{g}, \mathcal{J})$ be a Kaehler manifold and $\bar{\nabla}$ be an affine connection on $\overline{\mathcal{M}}$. Then $(\overline{\mathcal{M}}, \bar{\nabla}, \bar{g}, \mathcal{J})$ is said to be a holomorphic statistical manifold if

(a) $(\overline{\mathcal{M}}, \bar{\nabla}, \bar{g})$ is a statistical manifold, and

(b) a 2 -form $\varpi$ on $\overline{\mathcal{M}}$, given by $\varpi(\mathcal{X}, y)=\bar{g}(\mathcal{X}, \mathcal{\partial y})$ for any $\mathcal{X}, y \in \Gamma(T \overline{\mathcal{M}})$, is $\bar{\nabla}$-parallel, that is, $\bar{\nabla} \varpi=0$.

For a holomorphic statistical manifold $(\overline{\mathcal{M}}, \bar{g}, \mathcal{J})$, we have the following relation (see [12]) $\bar{\nabla}_{x}(\mathcal{J y})=\mathcal{\partial} \bar{\nabla}_{x}^{*} y$ for any $x, y \in \Gamma(T \overline{\mathcal{M}})$.

Lemma 2.1 ([11]). Let $(\overline{\mathcal{M}}, \bar{g}, \mathcal{J})$ be a Kaehler manifold and a connection $\bar{\nabla}$ is defined as $\bar{\nabla}:=\nabla^{\bar{g}}+K$, where $K$ is a $(1,2)$-tensor field satisfying the following conditions:

$$
\begin{aligned}
K(X, y) & =K(y, x), \\
\bar{g}(K(X, y), z) & =\bar{g}(y, K(X, z)),
\end{aligned}
$$

and $K(\mathcal{X}, \mathcal{\partial y})+\mathcal{J} K(\mathcal{X}, \mathcal{y})=0$ for any $X, y, z \in \Gamma(T \overline{\mathcal{M}})$. Then, $(\overline{\mathcal{M}}, \bar{\nabla}, \bar{g}, \mathcal{J})$ is a holomorphic statistical manifold.

By following [26] and Lemma 2.1, we have the following examples.

Example $2.2([26])$. Let $(\bar{g}, \mathcal{J})$ be a Kaehler structure on $\overline{\mathcal{M}}$. We take a vector field $U \in \Gamma(T \overline{\mathcal{M}})$ and set a tensor field $K_{1} \in \Gamma\left(T \overline{\mathcal{M}}^{(1,2)}\right)$ as follows:

$$
\begin{aligned}
K_{1}(x, y)= & {[\bar{g}(\mathcal{J} U, X) \bar{g}(\mathcal{J} U, y)-\bar{g}(U, x) \bar{g}(U, y)] U } \\
& +[\bar{g}(\mathcal{J} U, \mathcal{X}) \bar{g}(U, y)+\bar{g}(U, X) \bar{g}(\mathcal{J} U, y)] \mathcal{J} U,
\end{aligned}
$$


for any $x, y \in \Gamma(T \overline{\mathcal{M}})$. Then, by simple computation, we see that $K_{1}$ satisfies three conditions of Lemma 2.1, and hence a holomorphic statistical manifold $\left(\overline{\mathcal{M}}, \bar{\nabla}:=\nabla^{\bar{g}}+K_{1}, \bar{g}, \mathcal{J}\right)$ is obtained.

Example 2.3 ([26]). For a Kaehler manifold $(\overline{\mathcal{M}}, \bar{g}, \mathcal{J})$, we take a vector field $U \in \Gamma(T \overline{\mathcal{M}})$ and set $K_{2}$ as follows:

$$
\begin{aligned}
K_{2}(X, y)= & {[\bar{g}(U, \mathcal{X}) \bar{g}(U, \partial y)-\bar{g}(U, X) \bar{g}(U, y)} \\
& -\bar{g}(U, \partial \mathcal{X}) \bar{g}(U, y)-\bar{g}(U, X) \bar{g}(U, \partial y)] U \\
& +[\bar{g}(U, X) \bar{g}(U, y)-\bar{g}(U, \partial \mathcal{X}) \bar{g}(U, \partial y) \\
& -\bar{g}(U, \partial X) \bar{g}(U, y)-\bar{g}(U, X) \bar{g}(U, \partial y)] \partial U,
\end{aligned}
$$

for any $x, y \in \Gamma(T \overline{\mathcal{M}})$. Then $K_{2} \in \Gamma\left(T \overline{\mathcal{M}}^{(1,2)}\right)$ satisfies three conditions of Lemma 2.1 as in Example 2.2, and hence $\left(\overline{\mathcal{M}}, \bar{\nabla}:=\nabla^{\bar{g}}+K_{2}, \bar{g}, \mathcal{J}\right)$ becomes a holomorphic statistical manifold.

Example 2.4 ([26]). Let us consider a Kaehler manifold

$$
\left(\overline{\mathcal{M}}=\left\{\left(u^{1}, u^{2}\right)^{\prime} \in \mathbb{R}^{2} \mid u^{1}>0\right\}, \bar{g}, \mathcal{J}\right),
$$

where a Riemanian metric $\bar{g}$ and the standard complex structure $\mathcal{J}$ on $\overline{\mathcal{M}}$ are defined by $\bar{g}=u^{1}\left\{\left(d u^{1}\right)^{2}+\left(d u^{2}\right)^{2}\right\}$ and $\mathcal{\partial} \partial_{1}=\partial_{2}, \mathcal{\partial} \partial_{2}=-\partial_{1}$, where $\partial_{i}=\frac{\partial}{\partial u^{i}}$ for $i=1,2$. Now, for any $\kappa \in \mathbb{R}$, we define a $(1,2)$-tensor field $K_{3}$ on $\mathbb{R}^{2}$ as follows:

$$
K_{3}=\sum_{i, j, l=1}^{2} k_{i j}^{l} \partial_{l} \otimes d u^{i} \otimes d u^{j},
$$

where $-k_{11}^{1}=k_{12}^{2}=k_{21}^{2}=k_{22}^{1}=\kappa$ and $k_{11}^{2}=k_{12}^{1}=k_{21}^{1}=k_{22}^{2}=0$. Then $K_{3}$ satisfies all three conditions of Lemma 2.1, and hence we get a holomorphic statistical manifold $\left(\overline{\mathcal{M}}, \bar{\nabla}:=\nabla^{\bar{g}}+K_{3}, \bar{g}, \mathcal{J}\right)$, where an affine connection $\bar{\nabla}$ on $\overline{\mathcal{M}}$ is given by

$$
\begin{aligned}
& \bar{\nabla}_{\partial_{1}} \partial_{1}=\left(\frac{1}{2}\left(u^{1}\right)^{-1}-\kappa\right) \partial_{1}, \\
& \bar{\nabla}_{\partial_{1}} \partial_{2}=\bar{\nabla}_{\partial_{2}} \partial_{1}=\left(\frac{1}{2}\left(u^{1}\right)^{-1}+\kappa\right) \partial_{2}, \\
& \bar{\nabla}_{\partial_{2}} \partial_{2}=-\left(\frac{1}{2}\left(u^{1}\right)^{-1}-\kappa\right) \partial_{1} .
\end{aligned}
$$

Now, we pay attention to the concept of statistical hypersurface. Let $(\mathcal{M}, g)$ be a statistical hypersurface of a holomorphic statistical manifold $(\overline{\mathcal{M}}, \bar{g}, \mathcal{J})$. By the Kaehler structure $\mathcal{J}$, one can transfer any tangent vector field $\mathcal{X}$ on $\mathcal{M}$ in $\overline{\mathcal{M}}$ as follows: $\mathcal{J X}=\mathcal{P} \mathcal{X}+u(\mathcal{X}) \mathcal{N}$, where $\mathcal{P} \mathcal{X}=\tan (\mathcal{J X})$ and $\mathcal{N}$ is a unit normal vector field on $\mathcal{M}$ in $\overline{\mathcal{M}}$. 
Then, it naturally satisfies the following relations (see [12]):

$$
\left\{\begin{array}{l}
\mathcal{P}^{2} \mathcal{X}=-X+u(X) \xi \\
u(\xi)=1 \\
\mathcal{P} \xi=0
\end{array}\right.
$$

The fundamental equations in the geometry of Riemannian submanifolds are the Gauss and Weingarten formulae and the equations of Gauss, Codazzi and Ricci (see [29]). In the statistical setting, Gauss and Weingarten formulae are, respectively, defined by $[12]$

$$
\left\{\begin{array}{rlrl}
\bar{\nabla}_{x} y & =\nabla_{x} y+\varsigma(x, y) \mathcal{N}, & & \bar{\nabla}_{x}^{*} y=\nabla_{x}^{*} y+\varsigma^{*}(x, y) \mathcal{N} \\
\bar{\nabla}_{x} \mathcal{N}=-\Lambda(x)+\nu(x) \mathcal{N}, & & \bar{\nabla}_{x}^{*} \mathcal{N}=-\Lambda^{*}(x)+\nu^{*}(X) \mathcal{N}
\end{array}\right.
$$

for any $\mathcal{X}, y \in \Gamma(T \mathcal{M})$ and $\mathcal{N} \in \Gamma\left(T^{\perp} \mathcal{M}\right)$, where $\bar{\nabla}$ and $\bar{\nabla}^{*}$ (resp. $\nabla$ and $\nabla^{*}$ ) are the dual connections on $\overline{\mathcal{M}}$ (resp. on $\mathcal{M}$ ). Define $\nu$ and $\nu^{*}$ by $\nu(X)=g\left(D_{x} \mathcal{N}, \mathcal{N}\right)$ and $\nu^{*}(\mathcal{X})=g\left(D_{\mathcal{X}}^{*} \mathcal{N}, \mathcal{N}\right)$, respectively. The symmetric and bilinear imbedding curvature tensors of $\mathcal{M}$ in $\overline{\mathcal{M}}$ for $\bar{\nabla}$ and $\bar{\nabla}^{*}$ are denoted by $\varsigma$ and $\varsigma^{*}$, respectively. The relation between $\varsigma$ (resp., $\varsigma^{*}$ ) and $\Lambda$ (resp. $\Lambda^{*}$ ) is defined by [12]

$$
\left\{\begin{aligned}
\bar{g}(\varsigma(\mathcal{X}, \boldsymbol{y}), \mathcal{N}) & =g\left(\Lambda^{*} \mathcal{X}, y\right) \\
\bar{g}\left(\varsigma^{*}(\mathcal{X}, \boldsymbol{y}), \mathcal{N}\right) & =g(\Lambda \mathcal{X}, \boldsymbol{y})
\end{aligned}\right.
$$

for any $x, y \in \Gamma(T \mathcal{M})$ and $\mathcal{N} \in \Gamma\left(T^{\perp} \mathcal{M}\right)$.

Definition $2.4([5])$. Let $(\mathcal{M}, \nabla, g)$ be a submanifold with any codimension of a statistical manifold $(\overline{\mathcal{M}}, \bar{\nabla}, \bar{g})$. Then $\mathcal{M}$ is said to be

(a) totally geodesic with respect to $\bar{\nabla}$ if $\varsigma=0$;

(a) totally geodesic with respect to $\bar{\nabla}^{*}$ if $\varsigma^{*}=0$;

(b) tangentially totally umbilical with respect to $\bar{\nabla}$ if $\varsigma(\mathcal{X}, \mathcal{y})=g(\mathcal{X}, \mathcal{Y}) \mathcal{H}$ for any $\mathcal{X}, y \in \Gamma(T \mathcal{M})$, (here $\mathcal{H}$ is the mean curvature vector of $\mathcal{M}$ in $\overline{\mathcal{M}}$ for $\bar{\nabla}$ );

(b)* tangentially totally umbilical with respect to $\bar{\nabla}^{*}$ if $\varsigma^{*}(\mathcal{X}, \mathcal{Y})=g(\mathcal{X}, \mathcal{Y}) \mathcal{H}^{*}$ for any $\mathcal{X}, y \in \Gamma(T \mathcal{M})$, (here $\mathcal{H}^{*}$ is the mean curvature vector of $\mathcal{M}$ in $\overline{\mathcal{M}}$ for $\bar{\nabla}^{*}$ );

(c) normally totally umbilical with respect to $\bar{\nabla}$ if $\Lambda_{\mathcal{N}} \mathcal{X}=g(\mathcal{H}, \mathcal{N}) \mathcal{X}$ for any $\mathcal{X} \in \Gamma(T \mathcal{M})$ and $\mathcal{N} \in \Gamma\left(T^{\perp} \mathcal{M}\right)$

$(\mathrm{c})^{*}$ normally totally umbilical with respect to $\bar{\nabla}^{*}$ if $\Lambda_{\mathcal{N}}^{*} \mathcal{X}=g\left(\mathcal{H}^{*}, \mathcal{N}\right) \mathcal{X}$ for any $\mathcal{X} \in \Gamma(T \mathcal{M})$ and $\mathcal{N} \in \Gamma\left(T^{\perp} \mathcal{M}\right)$.

The curvature tensors with respect to $\bar{\nabla}$ and $\bar{\nabla}^{*}$ are denoted by $\overline{\mathcal{R}}$ and $\overline{\mathcal{R}}^{*}$, respectively. Also, $\mathcal{R}$ and $\mathcal{R}^{*}$ are the curvature tensors with respect to $\nabla$ and $\nabla^{*}$, respectively. Then the curvature tensor fields of $\overline{\mathcal{M}}$ and $\mathcal{M}$ are respectively defined as (see [12]) $\overline{\mathcal{S}}=\frac{1}{2}\left(\overline{\mathcal{R}}+\overline{\mathcal{R}}^{*}\right)$ and $\mathcal{S}=\frac{1}{2}\left(\mathcal{R}+\mathcal{R}^{*}\right)$.

The sectional curvature $\mathbb{K}$ on $\mathcal{M}$ of $\overline{\mathcal{M}}$ is given by (see [21,22])

$$
\mathbb{K}(X \wedge y)=g(\mathcal{S}(x, y) y, X)=\frac{1}{2}\left(g(\mathcal{R}(X, y) y, x)+g\left(\mathcal{R}^{*}(x, y) y, X\right)\right),
$$

for any orthonormal vectors $\mathcal{X}, y \in T_{\wp} \mathcal{M}, \wp \in \mathcal{M}$. 
Definition 2.5 ([12]). A holomorphic statistical manifold $(\overline{\mathcal{M}}, \bar{\nabla}, \bar{g}, \mathcal{J})$ is said to be of constant holomorphic curvature $k \in \mathbb{R}$ if the following curvature equation holds

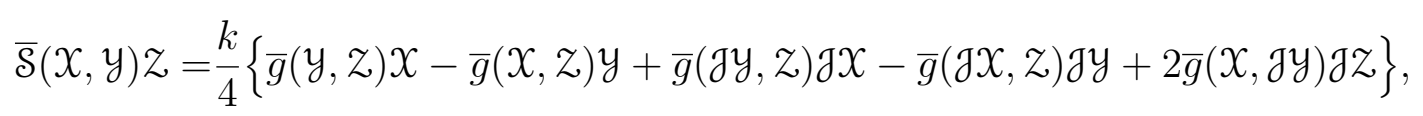

for any $x, y, z \in \Gamma(T \overline{\mathcal{M}})$. It is denoted by $\overline{\mathcal{M}}(k)$.

The corresponding Gauss equation is given by (see [12])

$$
\begin{aligned}
& \frac{k}{2}\{g(y, z) X-g(X, z) y+g(\mathcal{P} y, z) \mathcal{P} X-g(\mathcal{P} X, z) \mathcal{P} y+2 g(X, \mathcal{P} y) \mathcal{P} z\} \\
= & 2 \overline{\mathcal{S}}(X, y) z \\
= & 2 \mathcal{S}(X, y) z-g\left(\Lambda^{*} y, z\right) \Lambda X+g\left(\Lambda^{*} X, z\right) \Lambda y-g(\Lambda y, z) \Lambda^{*} X+g(\Lambda X, z) \Lambda^{*} y,
\end{aligned}
$$

for any $x, y, z \in \Gamma(T \mathcal{M})$.

\section{Casorati Curvatures for Statistical Hypersurfaces}

In this section, we study Casorati curvatures for a statistical hypersurface $\mathcal{M}$ of a holomorphic statistical manifold $\overline{\mathcal{M}}$.

We put $\operatorname{dim}(\mathcal{M})=m=2 n-1$ and $\operatorname{dim}(\overline{\mathcal{M}})=2 n$. Now, we consider a local orthonormal tangent frame $\left\{\mathcal{E}_{1}, \ldots, \mathcal{E}_{m}\right\}$ of $T \mathcal{M M}^{m}$ and a local orthonormal normal frame $\{\varepsilon\}$ of $T^{\perp} \mathcal{M}^{m}$ in $\overline{\mathcal{M}}^{2 n}$. The scalar curvature $\sigma(\wp)$ of $\mathcal{M}, \wp \in \mathcal{M}$, is given by

$$
\begin{aligned}
\sigma(\wp) & =\sum_{1 \leq i<j \leq m} g\left(\mathcal{S}\left(\mathcal{E}_{i}, \mathcal{E}_{j}\right) \mathcal{E}_{j}, \mathcal{E}_{i}\right) \\
& =\frac{1}{2}\left\{\sum_{1 \leq i<j \leq m} g\left(\mathcal{R}\left(\mathcal{E}_{i}, \mathcal{E}_{j}\right) \mathcal{E}_{j}, \mathcal{E}_{i}\right)+\sum_{1 \leq i<j \leq m} g\left(\mathcal{R}^{*}\left(\mathcal{E}_{i}, \mathcal{E}_{j}\right) \mathcal{E}_{j}, \mathcal{E}_{i}\right)\right\},
\end{aligned}
$$

and the normalized scalar curvature $\varrho$ of $\mathcal{M}$ is defined as

$$
\varrho=\frac{2 \sigma(\wp)}{m(m-1)} \text {. }
$$

The mean curvature vectors $\mathcal{H}$ and $\mathcal{H}^{*}$ of $\mathcal{M}$ in $\overline{\mathcal{M}}$ are given by

$$
\mathcal{H}=\frac{1}{m} \sum_{i=1}^{m} \varsigma\left(\mathcal{E}_{i}, \mathcal{E}_{i}\right), \quad\left(\text { resp. } \mathcal{H}^{*}=\frac{1}{m} \sum_{i=1}^{m} \varsigma^{*}\left(\mathcal{E}_{i}, \mathcal{E}_{i}\right)\right) .
$$

Conveniently, let us put

$$
\varsigma_{i j}=g\left(\varsigma\left(\mathcal{E}_{i}, \mathcal{E}_{j}\right), \mathcal{E}\right), \quad\left(\operatorname{resp} . \varsigma_{i j}^{*}=g\left(\varsigma^{*}\left(\mathcal{E}_{i}, \mathcal{E}_{j}\right), \mathcal{E}\right)\right),
$$

for $i, j=1, \ldots, m$.

Then, the squared norm of mean curvature vectors of $\mathcal{M}$ is defined as

$$
\|\mathcal{H}\|^{2}=\left(\frac{1}{m} \sum_{i=1}^{m} \varsigma_{i i}\right)^{2}, \quad\left(\text { resp. }\left\|\mathcal{H}^{*}\right\|^{2}=\left(\frac{1}{m} \sum_{i=1}^{m} \varsigma_{i i}^{*}\right)^{2}\right) .
$$


The squared norm of second fundamental forms $\varsigma$ and $\varsigma^{*}$ are denoted by $\mathcal{C}$ and $\mathcal{C}^{*}$, respectively, called the Casorati curvatures of $\mathcal{M}$ in $\overline{\mathcal{M}}$. Therefore, we have

$$
\mathcal{C}=\frac{1}{m}\|\varsigma\|^{2}, \quad\left(\text { resp. } \mathcal{C}^{*}=\frac{1}{m}\left\|\varsigma^{*}\right\|^{2}\right),
$$

where

$$
\|\varsigma\|^{2}=\sum_{i, j=1}^{m}\left(\varsigma_{i j}\right)^{2}, \quad\left(\operatorname{resp} .\left\|\varsigma^{*}\right\|^{2}=\sum_{i, j=1}^{m}\left(\varsigma_{i j}^{*}\right)^{2}\right) .
$$

If we consider a $r$-dimensional subspace $\mathcal{W}$ of $T \mathcal{M}, r \geq 2$, and an orthonormal basis $\left\{\mathcal{E}_{1}, \ldots, \mathcal{E}_{r}\right\}$ of $\mathcal{W}$. Then the scalar curvature of the $r$-plane section $\mathcal{W}$ is defined as

$$
\begin{aligned}
\sigma(\mathcal{W}) & =\sum_{1 \leq i<j \leq r} \mathcal{S}\left(\mathcal{E}_{i}, \mathcal{E}_{j}, \mathcal{E}_{j}, \mathcal{E}_{i}\right) \\
& =\frac{1}{2}\left\{\sum_{1 \leq i<j \leq r} \mathcal{R}\left(\mathcal{E}_{i}, \mathcal{E}_{j}, \mathcal{E}_{j}, \mathcal{E}_{i}\right)+\sum_{1 \leq i<j \leq r} \mathcal{R}^{*}\left(\mathcal{E}_{i}, \mathcal{E}_{j}, \mathcal{E}_{j}, \mathcal{E}_{i}\right)\right\},
\end{aligned}
$$

and the Casorati curvatures of the subspace $\mathcal{W}$ are the following:

$$
\mathcal{C}(\mathcal{W})=\frac{1}{r} \sum_{i, j=1}^{r}\left(\varsigma_{i j}\right)^{2}, \quad\left(\operatorname{resp} . \mathcal{C}^{*}(\mathcal{W})=\frac{1}{r} \sum_{i, j=1}^{r}\left(\varsigma_{i j}^{*}\right)^{2}\right) .
$$

The normalized Casorati curvatures $\delta_{\mathfrak{e}}(m-1)$ and $\widehat{\delta}_{\mathfrak{e}}(m-1)$ are defined as

(a)

$$
\begin{gathered}
{\left[\delta_{\mathcal{C}}(m-1)\right]_{\wp}=\frac{1}{2} \mathcal{C}_{\wp}+\left(\frac{m+1}{2 m}\right) \inf \left\{\mathfrak{C}(\mathcal{W}) \mid \mathcal{W}: \text { a hyperplane of } T_{\wp} \mathcal{M}\right\}} \\
\left(\text { resp. }\left[\delta_{\mathcal{C}}^{*}(m-1)\right]_{\wp}=\frac{1}{2} \mathcal{C}_{\wp}^{*}+\left(\frac{m+1}{2 m}\right) \inf \left\{\mathcal{C}^{*}(\mathcal{W}) \mid \mathcal{W}: \text { a hyperplane of } T_{\wp} \mathcal{M}\right\}\right)
\end{gathered}
$$

(b)

$$
\begin{gathered}
{\left[\widehat{\delta}_{\mathcal{C}}(m-1)\right]_{\wp}=2 \mathcal{C}_{\wp}-\left(\frac{2 m-1}{2 m}\right) \sup \left\{\mathcal{C}(\mathcal{W}) \mid \mathcal{W}: \text { a hyperplane of } T_{\wp} \mathcal{M} \mathcal{H}\right\}} \\
\left(\operatorname{resp.}\left[\widehat{\delta}_{\mathcal{C}}^{*}(m-1)\right]_{\wp}=2 \mathcal{C}_{\wp}^{*}-\left(\frac{2 m-1}{2 m}\right) \sup \left\{\mathcal{C}^{*}(\mathcal{W}) \mid \mathcal{W}: \text { a hyperplane of } T_{\wp} \mathcal{M}\right\}\right) .
\end{gathered}
$$

Further, we define the generalized normalized Casorati curvatures $\delta_{\mathfrak{e}}(s ; m-1)$ and $\widehat{\delta}_{\mathfrak{C}}(s ; m-1)$ as follows

(a) for $0<s<m^{2}-m$

$$
\left[\delta_{\mathfrak{C}}(s ; m-1)\right]_{\wp}=s \mathcal{C}_{\wp}+\zeta(s) \inf \left\{\mathfrak{C}(\mathcal{W}) \mid \mathcal{W}: \text { a hyperplane of } T_{\wp} \mathcal{M}\right\}
$$

(resp. $\left[\delta_{\mathcal{C}}^{*}(s ; m-1)\right]_{\wp}=s \mathcal{C}_{\wp}^{*}+\zeta(s) \inf \left\{\mathcal{C}^{*}(\mathcal{W}) \mid \mathcal{W}:\right.$ a hyperplane of $\left.\left.T_{\wp} \mathcal{M}\right\}\right)$; 
(b) for $s>m^{2}-m$

$$
\left[\widehat{\delta}_{\mathcal{C}}(s ; m-1)\right]_{\wp}=s \mathcal{C}_{\wp}+\zeta(s) \sup \left\{\mathfrak{C}(\mathcal{W}) \mid \mathcal{W}: \text { a hyperplane of } T_{\wp} \mathcal{M}\right\}
$$

$\left(\right.$ resp. $\left[\widehat{\delta}_{\mathcal{C}}^{*}(s ; m-1)\right]_{\wp}=s \mathcal{C}_{\wp}^{*}+\zeta(s) \sup \left\{\mathcal{C}^{*}(\mathcal{W}) \mid \mathcal{W}:\right.$ a hyperplane of $\left.\left.T_{\wp} \mathcal{M}\right\}\right)$, where $\zeta(s)=\frac{1}{s m}(m-1)(m+s)\left(m^{2}-m-s\right), s \neq m(m-1)$.

Throughout this paper, we work with the above mentioned notations only.

\section{Bounds of Normalized Scalar Curvature}

The most fascinating problem in the theory of Riemannian submanifolds is to find simple relationships between various invariants (intrinsic and extrinsic) of the submanifolds and Riemannian manifolds. Initially, B.-Y. Chen [7] obtained sharp optimal inequalities involving the intrinsic $\delta$-curvatures of Chen and the extrinsic squared mean curvature of submanifolds in a real space form. On the other hand, the study of $\delta$-Casorati [9] curvatures proposed new solutions to the above problem. In this section, we prove such inequalities for a statistical hypersurface $\left(\mathcal{M}^{m}, \nabla, g\right)$ of a holomorphic statistical manifold $\left(\overline{\mathcal{M}}^{2 n}, \bar{\nabla}, \bar{g}, \mathcal{J}\right)$ with constant holomorphic sectional curvature $k, \overline{\mathcal{M}}^{2 n}(k)$.

Theorem 4.1. Let $\mathcal{N}^{m}(m=2 n-1)$ be a statistical hypersurface of a $2 n$-dimesnional holomorphic statistical manifold with constant holomorphic sectional curvature $k$, $\overline{\mathcal{M}}^{2 n}(k)$. Then

$$
\varrho \geq \frac{k(m+3)}{4 m}+\frac{m}{m-1}\|\mathcal{H}\|\left\|\mathcal{H}^{*}\right\|-\frac{1}{m(m-1)}\|\varsigma\|\left\|\varsigma^{*}\right\| .
$$

Proof. Let an orthonormal frame of $\mathcal{M}$ be $\left\{\mathcal{E}_{1}, \ldots, \mathcal{E}_{m}\right\}$ and a unit normal vector to $\mathcal{M}$ be $\{\mathcal{E}\}$. From equation (2.2), we get

$$
2 \sigma=\frac{k(m+3)(m-1)}{4 m}+m^{2}\|\mathcal{H}\|\left\|\mathcal{H}^{*}\right\|-\sum_{i, j=1}^{m} \varsigma_{i j} \varsigma_{i j}^{*} .
$$

Applying Cauchy-Buniakowski-Schwarz, we have

$$
2 \sigma \geq \frac{k(m+3)(m-1)}{4 m}+m^{2}\|\mathcal{H}\|\left\|\mathcal{H}^{*}\right\|-\|\varsigma\|\left\|\varsigma^{*}\right\| .
$$

From last inequality, we can easily obtain (4.1). This is the required inequality.

Theorem 4.1 shows that the normalized scalar curvature is bounded below. Now, we switch to our next theorem, which shows that the normalized scalar curvature is bounded above in terms of Casorati curvature. The result is as follows.

Theorem 4.2. Let $\mathcal{M}^{m}(m=2 n-1)$ be a statistical hypersurface of a $2 n$-dimensional holomorphic statistical manifold with constant holomorphic sectional curvature $k$, $\overline{\mathcal{M}}^{2 n}(k)$. Then 
(a) the generalized normalized Casorati curvatures $\delta_{\mathfrak{C}}(s ; m-1)$ and $\delta_{\mathcal{C}}^{*}(s ; m-1)$ satisfy

$$
\varrho \leq \frac{2 \delta_{\mathcal{C}}^{0}(s ; m-1)}{m(m-1)}+\left[\frac{k(m+3)}{4 m}+\frac{\mathcal{C}^{0}}{m-1}-\frac{2 m}{m-1}\left\|\mathcal{H}^{0}\right\|^{2}+\frac{m}{m-1} g\left(\mathcal{H}, \mathcal{H}^{*}\right)\right],
$$

for any $s \in \mathbb{R}$ with $0<s<m(m-1)$, where $2 \mathcal{C}^{0}=\mathcal{C}+\mathcal{C}^{*}$ and $2 \delta_{\mathcal{C}}^{0}(s ; m-1)=$ $\delta_{\mathcal{e}}(s ; m-1)+\delta_{\mathcal{C}}^{*}(s ; m-1)$;

(b) the generalized normalized Casorati curvatures $\widehat{\delta}_{\mathfrak{C}}(s ; m-1)$ and $\widehat{\delta}_{\mathcal{C}}^{*}(s ; m-1)$ satisfy

$$
\varrho \leq \frac{2 \widehat{\delta}_{\mathcal{C}}^{0}(s ; m-1)}{m(m-1)}+\left[\frac{k(m+3)}{4 m}+\frac{\mathcal{C}^{0}}{m-1}-\frac{2 m}{m-1}\left\|\mathcal{H}^{0}\right\|^{2}+\frac{m}{m-1} g\left(\mathcal{H}, \mathcal{H}^{*}\right)\right],
$$

for any $s \in \mathbb{R}$, with $s>m(m-1)$, where $2 \mathcal{C}^{0}=\mathcal{C}+\mathcal{C}^{*}$ and $2 \widehat{\delta}_{\mathfrak{C}}^{0}(s ; m-1)=$ $\widehat{\delta}_{\mathcal{C}}(s ; m-1)+\widehat{\delta}_{\mathcal{C}}^{*}(s ; m-1)$.

Proof. Let an orthonormal frame of $\mathcal{M}$ be $\left\{\mathcal{E}_{1}, \ldots, \mathcal{E}_{m}\right\}$ and a unit normal vector to $\mathcal{M}$ be $\{\mathcal{E}\}$. From equation (2.2), we get

$$
\begin{aligned}
2 \sigma(\wp)= & \frac{k}{4}[(m-1)(m+3)]+2 m^{2}\left\|\mathcal{H}^{0}\right\|^{2}-\frac{m^{2}}{2}\left(\|\mathcal{H}\|^{2}+\left\|\mathcal{H}^{*}\right\|^{2}\right) \\
& -2 m \mathcal{C}^{0}+\frac{m}{2}\left(\mathcal{C}+\mathcal{C}^{*}\right) .
\end{aligned}
$$

Let us take a quadratic polynomial $\mathcal{K}$ in the components of the second fundamental form

$$
\begin{aligned}
\mathcal{K}= & s \mathcal{C}^{0}+\zeta(s) \mathcal{C}^{0}(\mathcal{W})-2 \sigma(\wp)+\frac{k}{4}[(m-1)(m+3)] \\
& -\frac{m^{2}}{2}\left(\|\mathcal{H}\|^{2}+\left\|\mathcal{H}^{*}\right\|^{2}\right)+\frac{m}{2}\left(\mathcal{C}+\mathcal{C}^{*}\right) .
\end{aligned}
$$

Without loss of generality, we assume that $\mathcal{W}$ is spanned by $\mathcal{E}_{1}, \ldots, \mathcal{E}_{m}$ and together with (4.4), we find that

$$
\mathcal{K}=\frac{m+s}{m} \sum_{i, j=1}^{m}\left(\varsigma_{i j}^{0}\right)^{2}+\frac{\zeta(s)}{m-1} \sum_{i, j=1}^{m-1}\left(\varsigma_{i j}^{0}\right)^{2}-\left(\sum_{i=1}^{m} \varsigma_{i i}^{0}\right)^{2}
$$

or

$$
\begin{aligned}
\mathcal{K}= & \sum_{i=1}^{m-1}\left[q\left(\varsigma_{i i}^{0}\right)^{2}+\frac{2(m+s)}{m}\left(\varsigma_{i m}^{0}\right)^{2}\right] \\
& +\left[2 q \sum_{1 \leq i \neq j \leq m-1}\left(\varsigma_{i j}^{0}\right)^{2}-2 \sum_{1 \leq i \neq j \leq m}\left(\varsigma_{i i}^{0} \varsigma_{j j}^{0}\right)+\frac{s}{m}\left(\varsigma_{m m}^{0}\right)^{2}\right],
\end{aligned}
$$

where

$$
q=\left(\frac{m+s}{m}+\frac{\zeta(s)}{m-1}\right)
$$


From (4.5), we observe that the solutions of the following system of linear homogenous equations:

$$
\left\{\begin{array}{l}
\frac{\partial \mathcal{K}}{\partial \varsigma_{i i}^{0}}=2 q\left(\varsigma_{i i}^{0}\right)-2 \sum_{l=1}^{m} \varsigma_{l l}^{0}=0 \\
\frac{\partial \mathcal{K}}{\partial \varsigma_{m m}^{0}}=\frac{2 s}{m} \varsigma_{m m}^{0}-2 \sum_{l=1}^{m-1} \varsigma_{l l}^{0}=0 \\
\frac{\partial \mathcal{K}}{\partial \varsigma_{i j}^{0}}=4 q \varsigma_{i j}^{0}=0 \\
\frac{\partial \mathcal{K}}{\partial \varsigma_{i m}^{0}}=4\left(\frac{m+s}{m}\right) \varsigma_{i m}^{0}=0
\end{array}\right.
$$

are the critical points

$$
\varsigma^{0 c}=\left(\varsigma_{11}^{0}, \varsigma_{12}^{0}, \ldots, \varsigma_{m m}^{0}\right)
$$

of $\mathcal{K}$, where $i, j=1, \ldots, m-1, i \neq j$.

Hence, every solution $\varsigma^{0 c}$ has $\varsigma_{i j}^{0}=0$ for $i \neq j$ and the determinant which corresponds to the first two equations of the above system is zero. Furthermore, the Hessian matrix $\mathcal{H} e s s_{\mathcal{K}}$ of $\mathcal{K}$ is given by

$$
\mathcal{H}_{\text {ess }}=\left(\begin{array}{ccc}
I & O & O \\
O & I I & O \\
O & O & \text { III }
\end{array}\right),
$$

where $O$ are the null matrices and the matrices $I, I I$ and $I I I$ are, respectively, given below:

$$
\begin{aligned}
& I=-2\left(\begin{array}{cccccc}
1-q & 1 & \ldots & 1 & 1 \\
1 & 1-q & \ldots & 1 & 1 \\
\vdots & \vdots & \ddots & \vdots & \vdots \\
1 & & 1 & \ldots & 1-q & 1 \\
1 & & 1 & \ldots & 1 & \frac{-s}{m}
\end{array}\right) \\
& I I=4 q\left(\begin{array}{ccccc}
1 & 0 & \ldots & 0 & 0 \\
0 & 1 & \ldots & 0 & 0 \\
\vdots & \vdots & \ddots & \vdots & \vdots \\
0 & 0 & \ldots & 1 & 0 \\
0 & 0 & \ldots & 0 & 1
\end{array}\right), \\
& I I I=\frac{4(m+s)}{m}\left(\begin{array}{ccccc}
1 & 0 & \ldots & 0 & 0 \\
0 & 1 & \ldots & 0 & 0 \\
\vdots & \vdots & \ddots & \vdots & \vdots \\
0 & 0 & \ldots & 1 & 0 \\
0 & 0 & \ldots & 0 & 1
\end{array}\right) .
\end{aligned}
$$


Therefore, the eigenvalues of Hessian matrix $\mathcal{H} e s s_{\mathcal{K}}$ are given below:

$$
\begin{aligned}
& \mu_{11}=0, \mu_{22}=2\left(\frac{2 s}{m}+\frac{\zeta(s)}{m-1}\right), \quad \mu_{33}=\cdots=\mu_{m m}=2 q, \\
& \mu_{i j}=4 q, \mu_{i m}=\frac{4(m+s)}{m}, \quad \text { for all } i, j=1,2, \ldots, m-1, i \neq j .
\end{aligned}
$$

Thus, we know that $\mathcal{K}$ is parabolic and reaches a minimum $\mathcal{K}\left(\varsigma^{0 c}\right)$ for each solution $\varsigma^{0 c}$ of the system (4.6). From the equations (4.5) and (4.6), we arrive at $\mathcal{K}\left(\varsigma^{0 c}\right)=0$. Hence $\mathcal{K} \geq 0$, and this further gives following inequality:

$$
\begin{aligned}
2 \sigma(\wp) \leq & s \mathrm{C}^{0}+\zeta(s) \mathfrak{C}^{0}(\mathcal{W})+\frac{k(m-1)(m+3)}{4} \\
& -\frac{m^{2}}{2}\left(\|\mathcal{H}\|^{2}+\left\|\mathcal{H}^{*}\right\|^{2}\right)+\frac{m}{2}\left(\mathcal{C}+\mathcal{C}^{*}\right)
\end{aligned}
$$

Hence, we find that

$$
\begin{aligned}
\varrho \leq & \frac{s}{m(m-1)} \mathcal{C}^{0}+\frac{\zeta(s)}{m(m-1)} \mathrm{e}^{0}(\mathcal{W})+\frac{k(m+3)}{4 m} \\
& -\frac{2 m}{m-1}\left\|\mathcal{H}^{0}\right\|^{2}+\frac{m}{m-1} g\left(\mathcal{H}, \mathcal{H}^{*}\right)+\frac{1}{2(m-1)}\left(\mathcal{C}+\mathcal{C}^{*}\right),
\end{aligned}
$$

for every tangent hyperplane $\mathcal{W}$ of $\mathcal{M}$. If we take the infimum over all tangent hyperplanes $\mathcal{W}$, our assertion (4.2) follows.

In the same manner, we can establish an inequality (4.3) in the second part of the theorem.

Remark 4.1. The proof of Theorem 4.2 is mainly based on a classical optimization procedure by showing that a quadratic polynomial in the components of the second fundamental form $\varsigma^{0}$ with respect to Levi-Civita connection is parabolic (see $[15,16$, 18, 24, 27]). Since, we have proved that the Hessian matrix (4.8) is positive semidefinite for all points and admits precisely one eigenvalue equal to zero. Therefore, it is easy to say that $\mathcal{K}$ is parabolic and reaches a minimum $\mathcal{K}\left(\varsigma^{0 c}\right)$ for each solution $\varsigma^{0 c}$ of the system (4.6). In fact, because of the convexity, the critical point is a global minimum. We note that an alternative proof of Theorem 4.2 can be done by making use of T. Oprea's optimization technique [23], namely analyzing a suitable constrained extremum problem (see also $[8,19,25]$ ).

The characterisation of equality cases in Theorem 4.2.

Theorem 4.3. Let $\mathcal{N}^{m}(m=2 n-1)$ be a statistical hypersurface of a $2 n$-dimensional holomorphic statistical manifold with constant holomorphic sectional curvature $k$, $\overline{\mathcal{M}}^{2 n}(k)$. Equalities hold in the relations (4.2) and (4.3) if and only if

$$
\varsigma_{i j}=-\varsigma_{i j}^{*}, \quad \text { for all } i, j=1, \ldots, m, i \neq j,
$$


and

$$
\varsigma_{m m}^{0}=\frac{m(m-1)}{s} \varsigma_{11}^{0}=\cdots=\frac{m(m-1)}{s} \varsigma_{m-1 m-1}^{0} .
$$

\section{Some Geometric Applications}

In this section, we discuss some immediate applications of the results proved in the previous section. Some immediate consequences of Theorem 4.2 are the following.

Corollary 5.1. Let $\mathcal{M}^{m}(m=2 n-1)$ be a statistical hypersurface of a $2 n$-dimensional holomorphic statistical manifold with constant holomorphic sectional curvature $k$, $\overline{\mathcal{M}}^{2 n}(k)$. Then

(a) the normalized Casorati curvatures $\delta_{\mathfrak{C}}(m-1)$ and $\delta_{\mathcal{C}}^{*}(m-1)$ satisfies

$$
\varrho \leq 2 \delta_{\mathfrak{C}}^{0}(m-1)+\left[\frac{k(m+3)}{4 m}+\frac{\mathcal{C}^{0}}{m-1}-\frac{2 m}{m-1}\left\|\mathcal{H}^{0}\right\|^{2}+\frac{m}{m-1} g\left(\mathcal{H}, \mathcal{H}^{*}\right)\right],
$$

where $2 \mathfrak{C}^{0}=\mathcal{C}+\mathcal{C}^{*}$ and $2 \delta_{\mathfrak{C}}^{0}(m-1)=\delta_{\mathfrak{C}}(m-1)+\delta_{\mathfrak{C}}^{*}(m-1)$;

(b) the normalized Casorati curvatures $\widehat{\delta}_{\mathfrak{C}}(m-1)$ and $\widehat{\delta}_{\mathcal{C}}^{*}(m-1)$ satisfies

$$
\varrho \leq 2 \widehat{\delta}_{\mathfrak{C}}^{0}(m-1)+\left[\frac{k(m+3)}{4 m}+\frac{\mathcal{C}^{0}}{m-1}-\frac{2 m}{m-1}\left\|\mathcal{H}^{0}\right\|^{2}+\frac{m}{m-1} g\left(\mathcal{H}, \mathcal{H}^{*}\right)\right],
$$

where $2 \mathfrak{C}^{0}=\mathfrak{C}+\mathfrak{C}^{*}$ and $2 \widehat{\delta}_{\mathfrak{C}}^{0}(m-1)=\widehat{\delta}_{\mathfrak{C}}(m-1)+\widehat{\delta}_{\mathcal{C}}^{*}(m-1)$.

Remark 5.1. We remark that one can prove Corollary 5.1 by considering $s=\frac{m(m-1)}{2}$ in $\delta_{\mathcal{C}}(s ; m-1)\left(\operatorname{resp} . \delta_{\mathcal{C}}^{*}(s ; m-1)\right)$ and we have the following relation (see [16])

$$
\begin{aligned}
& {\left[\delta_{\mathcal{C}}\left(\frac{m(m-1)}{2} ; m-1\right)\right]_{\wp}=m(m-1)\left[\delta_{\mathfrak{C}}(m-1)\right]_{\wp} } \\
&\left(\operatorname{resp.}\left[\delta_{\mathfrak{C}}^{*}\left(\frac{m(m-1)}{2} ; m-1\right)\right]_{\wp}=m(m-1)\left[\delta_{\mathfrak{C}}^{*}(m-1)\right]_{\wp}\right)
\end{aligned}
$$

at any point $\wp \in \mathcal{M}$.

Corollary 5.2. Let $\mathcal{M}^{m}(m=2 n-1)$ be a statistical hypersurface of a $2 n$-dimensional holomorphic statistical manifold with constant holomorphic sectional curvature $k$, $\overline{\mathcal{M}}^{2 n}(k)$. If $\mathcal{M}$ is minimal, i.e., $\mathcal{H}^{0}=0$, then

(a) the generalized normalized Casorati curvatures $\delta_{\mathcal{C}}(s ; m-1)$ and $\delta_{\mathcal{C}}^{*}(s ; m-1)$ satisfy

$$
\varrho \leq 2 \frac{\delta_{\mathcal{\varrho}}^{0}(s ; m-1)}{m(m-1)}+\frac{k(m+3)}{4 m}+\frac{\mathcal{C}^{0}}{m-1}+\frac{m}{m-1} g\left(\mathcal{H}, \mathcal{H}^{*}\right),
$$

for any $s \in \mathbb{R}$, with $0<s<m(m-1)$, where $2 \mathfrak{C}^{0}=\mathcal{C}+\mathcal{C}^{*}$ and $2 \delta_{\mathcal{C}}^{0}(s ; m-1)=$ $\delta_{\mathfrak{e}}(s ; m-1)+\delta_{\mathfrak{C}}^{*}(s ; m-1)$; 
(b) the generalized normalized Casorati curvatures $\widehat{\delta}_{\mathfrak{e}}(s ; m-1)$ and $\widehat{\delta}_{\mathfrak{C}}^{*}(s ; m-1)$ satisfy

$$
\varrho \leq 2 \frac{\widehat{\delta}_{\mathcal{C}}^{0}(s ; m-1)}{m(m-1)}+\frac{k(m+3)}{4 m}+\frac{\mathcal{C}^{0}}{m-1}+\frac{m}{m-1} g\left(\mathcal{H}, \mathcal{H}^{*}\right),
$$

for any $s \in \mathbb{R}$, with $s>m(m-1)$, where $2 \mathcal{C}^{0}=\mathcal{C}+\mathcal{C}^{*}$ and $2 \widehat{\delta}_{\mathcal{C}}^{0}(s ; m-1)=$ $\widehat{\delta}_{\mathcal{C}}(s ; m-1)+\widehat{\delta}_{\mathcal{C}}^{*}(s ; m-1)$.

The following result follows directly from Corollary 5.1.

Corollary 5.3. Let $\mathcal{N}^{m}(m=2 n-1)$ be a statistical hypersurface of a $2 n$-dimensional holomorphic statistical manifold with constant holomorphic sectional curvature $k$, $\overline{\mathcal{M}}^{2 n}(k)$. If $\mathcal{M}$ is minimal, i.e., $\mathcal{H}^{0}=0$, then

(a) the normalized Casorati curvature $\delta_{\mathfrak{C}}(m-1)$ and $\delta_{\mathfrak{C}}^{*}(m-1)$ satisfy

$$
\varrho \leq 2 \delta_{\mathfrak{C}}^{0}(m-1)+\frac{k(m+3)}{4 m}+\frac{\varrho^{0}}{m-1}+\frac{m}{m-1} g\left(\mathcal{H}, \mathcal{H}^{*}\right),
$$

where $2 \mathfrak{C}^{0}=\mathfrak{C}+\mathfrak{C}^{*}$ and $2 \delta_{\mathfrak{C}}^{0}(m-1)=\delta_{\mathfrak{C}}(m-1)+\delta_{\mathcal{C}}^{*}(m-1)$;

(b) the normalized Casorati curvature $\widehat{\delta}_{\mathfrak{C}}(m-1)$ and $\widehat{\delta}_{\mathcal{C}}^{*}(m-1)$ satisfy

$$
\varrho \leq 2 \widehat{\delta}_{\mathfrak{e}}^{0}(m-1)+\frac{k(m+3)}{4 m}+\frac{\mathfrak{C}^{0}}{m-1}+\frac{m}{m-1} g\left(\mathcal{H}, \mathcal{H}^{*}\right),
$$

where $2 \mathfrak{C}^{0}=\mathcal{C}+\mathcal{C}^{*}$ and $2 \widehat{\delta}_{\mathfrak{C}}^{0}(m-1)=\widehat{\delta}_{\mathfrak{C}}(m-1)+\widehat{\delta}_{\mathfrak{C}}^{*}(m-1)$.

Now, we have the following statistical significance of Theorem 4.1.

Corollary 5.4. Let $\mathcal{M}^{m}(m=2 n-1)$ be a statistical hypersurface of a $2 n$-dimensional holomorphic statistical manifold with constant holomorphic sectional curvature $k$, $\overline{\mathcal{M}}^{2 n}(k)$. If $\mathcal{M}$ is totally umbilical and totally geodesic with respect to $\bar{\nabla}$ and $\bar{\nabla}^{*}$. Then

$$
\varrho \geq \frac{k(m+3)}{4 m} .
$$

Remark 5.2. In the above Corollary 5.4, we have $\mathcal{M}$ is totally umbilical and totally geodesic with respect to $\bar{\nabla}$ and $\bar{\nabla}^{*}$, that is, for any $\mathcal{X}, y \in T_{p} \mathcal{M}, 0=\varsigma(\mathcal{X}, \mathcal{y})=$ $g(\mathcal{X}, \boldsymbol{y}) \mathcal{H}$, which gives $\mathcal{H}=0$. Similarly, $0=\varsigma^{*}(\mathcal{X}, \boldsymbol{y})=g(\mathcal{X}, \boldsymbol{y}) \mathcal{H}^{*}$ implies $\mathcal{H}^{*}=0$. Hence, an inequality (4.1) reduces to (5.1).

Further, we observe the following.

Corollary 5.5. Let $\mathcal{M}^{m}(m=2 n-1)$ be a statistical hypersurface of a $2 n$-dimensional holomorphic statistical manifold with constant holomorphic sectional curvature $k$, $\overline{\mathcal{M}}^{2 n}(k)$. Suppose that $\varrho=\frac{k(m+3)}{4 m}$. Then $\mathcal{M}$ is not totally geodesic with respect to $\bar{\nabla}$ and $\bar{\nabla}^{*}$. 
Acknowledgements. The authors thank the referee for his/her valuable suggestions which helped to improve the paper.

\section{REFERENCES}

[1] S. Amari, Differential Geometric Methods in Statistics, Lecture Notes in Statistics 28, Springer, New York, 1985.

[2] M. Aquib and M. H. Shahid, Generalized normalized $\delta$-Casorati curvature for statistical submanifolds in quaternion Kahler-like statistical space forms, J. Geom. 109 (2018), 13 pages.

[3] M. E. Aydin, A. Mihai and I. Mihai, Some Inequalities on submanifolds in statistical manifolds of constant curvature, Filomat 29(3) (2015), 465-477.

[4] M. E. Aydin, A. Mihai and I. Mihai, Generalized Wintgen inequality for statistical submanifolds in statistical manifolds of constant curvature, Bull. Math. Sci. (2016), DOI 10.1007/s13373-0160086-1.

[5] M. N. Boyom, A. N. Siddiqui, W. A. Mior Othman and M. H. Shahid, Classification of totally umbilical CR-statistical submanifolds in holomorphic statistical manifolds with constant holomorphic curvature, in: F. Nielsen, F. Barbaresco (Eds.), Geometric Science of Information, Lecture Notes in Computer Science 10589, Springer, Cham, 2017.

[6] F. Casorati, Mesure de la courbure des surface suivant 1'idee commune. Ses rapports avec les mesures de coubure gaussienne et moyenne, Acta Math. 14, (1999), 95-110.

[7] B.-Y. Chen, Some pinching and classification theorems forminimal submanifolds, Arch. Math. 60 (1993), 569-578.

[8] S. Decu, S. Haesen, L. Verstraelen and G. E. Vilcu, Curvature invariants of statistical submanifolds in Kenmotsu statistical manifolds of constant $\phi$-sectional curvature, Entropy 20(7) (2018), 529.

[9] S. Decu, S. Haesen and L. Verstraelen, Optimal inequalities characterising quasi-umbilical submanifolds, Journal of Inequalities in Pure and Applied Mathematics 9(3) (2008), 7 pages.

[10] S. Decu, A. Pantic, M. Petrovic-Torgasev and L. Verstraelen, Ricci and Casorati principal directions of $\delta(2)$ Chen ideal submanifolds, Kragujevac J. Math. 37(1) (2013), 25-31.

[11] H. Furuhata, Hypersurfaces in statistical manifolds, Differential Geom. Appl. 27 (2009), 420-429.

[12] H. Furuhata and I. Hasegawa, Submanifold theory in holomorphic statistical manifolds, In: S. Dragomir, M. H. Shahid and F. R. Al-Solamy (Eds.), Geometry of Cauchy-Riemann Submanifolds, Springer, Singapore, 2016, 179-215.

[13] H. Furuhata, I. Hasegawa, Y. Okuyama, K. Sato and M. H. Shahid, Sasakian statistical manifolds, J. Geom. Phys. 117 (2017), 179-186.

[14] H. Furuhata, I. Hasegawa, Y. Okuyama and K. Sato, Kenmotsu statistical manifolds and warped product, J. Geom. (2017), DOI 10.1007/s00022-017-0403-1.

[15] C. W. Lee, J. W. Lee, G. E. Vilcu and D. W. Yoon, Optimal inequalities for the Casorati curvatures of the submanifolds of generalized space form endowed with semi-symmetric metric connections, Bull. Korean Math. Soc. 52 (2015), 1631-1647.

[16] C. W. Lee and G. E. Vilcu, Inequalities for generalized normalized $\delta$-Casorati curvatures of slant submanifolds in quaternion space forms, Taiwanese J. Math. 19 (2015), 691-702.

[17] C. W. Lee, D. W. Yoon and J. W. Lee, A pinching theorem for statistical manifolds with Casorati curvatures, J. Nonlinear Sci. Appl. 10 (2017), 4908-4914.

[18] C. W. Lee, D. W. Yoon and J. W. Lee, Optimal inequalities for the Casorati curvatures of submanifolds of real space forms endowed with semi-symmetric metric connections, J. Inequal. Appl. 2014 (2014), 9 pages.

[19] C. W. Lee, J. W. Lee and G. E. Vilcu, A new proof for some optimal inequalities involving generalized normalized $\delta$-Casorati curvatures, J. Inequal. Appl. 2015 (2015), 9 pages. 
[20] C. W. Lee and J. W. Lee, Inequalities on Sasakian statistical manifolds in terms of casorati curvatures, Mathematics 6(259) (2018), 10 pages.

[21] B. Opozda, Bochner's technique for statistical structures, Ann. Global Anal. Geom. 48(4) (2015), 357-395.

[22] B. Opozda, A sectional curvature for statistical structures, Linear Algebra Appl. 497 (2016), 134-161.

[23] T. Oprea, Optimization methods on Riemannian submanifolds, An. Univ. Bucur. Mat. 54(1) (2005), 127-136.

[24] A. N. Siddiqui and M. H. Shahid, A lower bound of normalized scalar curvature for bi-slant submanifolds in generalized Sasakian space forms using Casorati curvatures, Acta Math. Univ. Comenianae 87(1) (2018), 127-140.

[25] A. N. Siddiqui, Upper bound inequalities for $\delta$-Casorati curvatures of submanifolds in generalized Sasakian space forms admitting a semi-symmetric metric connection, Int. Electron. J. Geom. 11(1) (2018), 57-67.

[26] A. N. Siddiqui and M. H. Shahid, On totally real statistical submanifold, Filomat 32(13) (2018), 11 pages.

[27] V. Slesar, B. Sahin and G. E. Vilcu, Inequalities for the Casorati curvatures of slant submanifolds in quaternionic space forms, J. Inequal. Appl. 2014 (2014), 10 pages.

[28] A. D. Vilcu and G. E. Vilcu, Statistical manifolds with almost quaternionic structures and quaternionic Kähler-like statistical submersions, Entropy 17 (2015), 6213-6228.

[29] K. Yano and M. Kon, Structures on Manifolds, Worlds Scientific, Singapore, 1984.

${ }^{1}$ Department of Mathematics,

Faculty of Natural Sciences,

Jamia Millia Islamia,

New Delhi-110025, India

Email address: aliyanaazsiddiqui9@gmail.com

Email address: hasan_jmi@yahoo.com 